\title{
Field Persistence of Some Novel Insecticides Residues on Cotton Plants and Their Latent Effects against Spodoptera littoralis (Boisduval)
}

\author{
El-Zahi S. El-Zahi ${ }^{1}$
}

\begin{abstract}
New insecticides that exhibited high compatibility with environment components are very promising substitutes for control of the economic insects. Simi-field experiments were conducted on profenofos and four novel insecticides viz., spinosad, emamectin-benzoate, pyridalyl and indoxacarb to investigate their efficiency and the field persistence of their residues against $4^{\text {th }}$ instar larvae of the cotton leaf worm, Spodoptera littoralis (Boisd.), field strain via determination of $\mathrm{Lt}_{50}$ values. The recommended rates of these insecticides were sprayed on the foliage of cotton plants, var. Giza 86, in the field. Also, latent effects after feeding of larvae on sprayed leaves for $24 \mathrm{~h}$ were studied under laboratory conditions. Pyridalyl and emamectinbenzoate had significantly the most persistent residual activity with $\mathrm{Lt}_{50}$ values of 6.74 and 5.51 days, respectively. Whereas, indoxacarb, spinosad and profenofos displayed inferior persistence periods translated in $\mathbf{L t}_{50}$ values of $0.72,1.33$ and 1.55 days, respectively. The high efficiency of pyridalyl and emamectin-benzoate decreased gradually after spray causing $100,100,95,87$, and $72 \%$ larval mortality for pyridalyl and $100,92,88,66$, and $41 \%$ larval mortality for emamectin-benzoate after $0,1,2,4$, and 6 days of spray, respectively. Two days post spray, the toxic effect of profenofos declined sharply recording 94, 60, 45, 30 , and $10 \%$ larval mortality after $0,1,2,4$, and 6 days of application, respectively. As the time after spray extended, the latent effects of the tested insecticides on survived larvae and consequent stages significantly decreased. Pyridalyl and emamectin-benzoate resulted in the superior latent effects even at the $6^{\text {th }}$ day of spray; causing larval duration of $20.1 \& 19.6$ days, $20.4 \& 42.6 \%$ normal pupae and $26.7 \& 55.2 \%$ normal adult emergence, respectively comparing to the other treatments. These results indicated that novel insecticides had the potentiality to be promising substitutes of conventional toxicants for $S$. littoralis control under field conditions.
\end{abstract}

Key words: Cotton, Spodoptera littoralis, Novel insecticides, Field persistence, Residual activity, Latent effects.

\section{INTRODUCTION}

The Egyptian cotton leafworm, Spodoptera littoralis (Boisduval) (Lepidoptera: Noctuidae) is one of the most destructive and economic pests of cotton, vegetables, ornamentals and other field crops. This pest is widely distributed throughout Africa, Mediterranean Europe and several parts of Asia (Azab et al., 2001). Caterpillars of $S$. littoralis are very polyphagous and can feed on approximately 90 economically important plant species belonging to 40 families (Brown and Dewhurst, 1975). Application of chemical insecticides to control $S$. littoralis is unavoidable procedure especially when outbreak occurs or its population density exceeds the economic threshold. Over the past 25 years, the intensive and unwise application of broad-spectrum insecticides against $S$. littoralis led to development of its resistance to many registered toxicants for its control and caused serious problems in environment components and natural balance between pests and their natural enemies (Smagghe et al., 1999; Abo-Elghar et al., 2005; Aydin and Gürkan, 2006).

Scientists and growers are seeking alternative materials that are effective against $S$. littoralis, safe to untargeted organisms and environment components and compatible with integrated pest management practices. In this respect, using new types of insecticides that originated from natural agents or disrupt the physiological processes of the targeted pests could be useful as alternatives for conventional insecticides (Thompson et al., 2000; Smagghe et al., 2003; Pineda et al., 2007). Several investigations indicated that, novel insecticides including Oxadiazines, Avermectins, Spinosyns, and Pyridalyl which characterized with their new and/or unique modes of action; have the potential for crop protection against economic pests and low toxicity to environment components and natural enemies (Foster et al., 2003, Michaud and Grant, 2003; Sakamoto et al., 2005).

Insecticides persistence on sprayed foliages depends upon many factors: physiochemical properties of the insecticide and additive materials, chemistry $(\mathrm{pH}$ and hardness) of the spray water, environmental factors and surface chemistry of the sprayed plants (McDonald et al., 1998; Argentine et al., 2002; Abdu-Allah, 2010). Moreover, under the same weathering conditions, the persistence period of the insecticide residues on plant foliage significantly differs based on the kind of sprayed plant (Holland et al., 1984; McDonald et al., 1998) and the targeted insect (Ishaaya et al., 2002). Cotton growers are seeking insecticide residues to be effective against targeted pests for longest periods as possible to avoid treatments reiteration. The objective of this study was to determine the residues persistence periods $\left(\mathrm{Lt}_{50}\right)$ and latent effects (larval duration, pupation percentages and percentages of adult emergence) of profenofos and four

\footnotetext{
${ }^{1}$ Plant Protection Res. Institute, Agricultural Res. Center, Giza, Egypt Received January31, 2013, Accepted March14, 2013
} 
novel insecticides i.e. spinosad, emamectin-benzoate, pyridalyl and indoxacarb against field strain of $S$. littoralis under semi-field conditions.

\section{MATERIALS AND METHODS}

\subsection{Tested insecticides:}

The field recommended rates of the following insecticides were tested against $4^{\text {th }}$ instar larvae of $S$. littoralis using commercial formulation of each.

- Spinosyn insecticide, spinosad (Tracer, SC 24\%), obtained from Dow AgroSciences Co. and was applied at $60 \mathrm{mg} \mathrm{AI} / \mathrm{L}$.

- Oxadiazine insecticide, indoxacarb (Steward, EC 15\%), provided from Du Pont Co. and was applied at $39 \mathrm{mg} \mathrm{AI} / \mathrm{L}$.

- The novel insecticide, pyridalyl (Pleo, EC 50\%), supplied from Sumitomo Chemical Co. and was applied at $250 \mathrm{mg} \mathrm{AI} / \mathrm{L}$.

- Avermectin derivative insecticide, emamectinbenzoate (Proclaim, SG 5\%), obtained from Syngenta Co. and was applied at $15 \mathrm{mg} \mathrm{AI} / \mathrm{L}$.

- Organophosphate insecticide, profenofos (Adwiprof, EC 72\%), supplied from Adwia Co. and was applied at $2700 \mathrm{mg} \mathrm{AI} / \mathrm{L}$.

\subsection{Insect rearing:}

Egg masses of $S$. littoralis field strain were collected from cotton fields of Kafr El- Sheikh Region, which did not receive any insecticidal treatments before egg masses collection. The egg masses were transferred to the laboratory and maintained under conditions of $25 \pm$ $2^{\circ} \mathrm{C}, 65 \pm 5 \mathrm{RH}$ and 14:10, L: D, photoperiod even developed into $4^{\text {th }}$ instar larvae; then used in the test. The larvae were fed on fresh leaves of castor bean, Ricinus communis, as described by El-Defrawi et al. (1964).

\subsection{Application of the tested insecticides:}

Application of the tested insecticides was conducted at Sakha Agricultural Research Station farm, where an area of $2100 \mathrm{~m}^{2}$ was selected to be sown on $8^{\text {th }}$ of April, 2012 with cotton seeds var. Giza 86; received all good recommended agricultural practices without any insecticidal treatments. This area was divided into plots each of $42 \mathrm{~m}^{2}$. Six treatments, the five tested insecticides and the check, were designed in this area. Four plots (replications) were made for each treatment. The treatments were arranged in a randomized complete block design. Application of tested insecticides was done on $6^{\text {th }}$ of July, 2012 under field conditions of $35 \pm$ $2^{\circ} \mathrm{C}$ at day, $75 \pm 5 \mathrm{RH}$ and 14:10, L: D, photoperiod. Irrigation water was used in diluting of the tested insecticides at their field recommended rates, and the final volume of spray solution represented 476 liters per hectare. Knapsack sprayer $\left(\mathrm{CP}_{3}\right)$ equipped with one nozzle was used. Unplanted belts ( $3 \mathrm{~m}$ width) were left as barriers between plots to avoid contamination with drifts.

\subsection{Laboratory experiments:}

The experiments were performed under laboratory conditions of $25 \pm 2^{\circ} \mathrm{C}, 70 \pm 5 \mathrm{RH}$ and 14:10, L: D, photoperiod. Five $S$. littoralis $4^{\text {th }}$ instar larvae were put in a $500 \mathrm{ml}$ plastic pot and covered with a clean piece of muslin cloth, representing one replication. Ten replications were made for each treatment at each date of feeding. The sprayed cotton leaves were picked up immediately after one $\mathrm{h}$ from spray (zero time), and then after 1, 2, 4, and 6 days post spray and transferred directly to the laboratory for feeing the selected larvae. After $24 \mathrm{~h}$ of feeding on treated leaves, the survived larvae were transmitted to new and clean $500 \mathrm{ml}$ plastic pots and were fed on untreated cotton leaves till pupation. Number of dead larvae and percentage of mortality were recorded $48 \mathrm{~h}$ post treatment. The larva was considered dead if no movement was observed when it was touched with a small brush. Larval duration, percentages of normal and deformed pupae, and percentages of normal and malformed adult emergence were estimated.

\subsection{Statistical analysis:}

Mortality percentages were corrected for mortality in control using the formula of Abbott (1925) and analyzed by probit analysis (Finney, 1971) using POLO- PC software (Le Ora Software, 1987) for time-mortality regression lines. Differences were considered significant based upon non-overlapping of $95 \%$ fiducial limits. Percentages of corrected mortality, pupation and adult emergence were subjected to one-way ANOVA by SPSS 13.0 (SPSS, 2004). Duncan's Multiple Range Test (DMRT) was used to determine significant differences between larval duration means $(\mathrm{P}<0.05)$ by CoStat system for Windows, Version 6.311, Berkeley, CA, USA, CoStat Program (2006).

\section{RESULTS AND DISCUSSIONS}

The field recommended rates of profenofos and other four novel insecticides viz., spinosad, emamectinbenzoate, pyridalyl and indoxacarb were sprayed on cotton foliage under field conditions to study the persistence of their residues against $4^{\text {th }}$ instar larvae of $S$. littoralis via determination of $\mathrm{Lt}_{50}$ when the larvae were fed for $24 \mathrm{~h}$ on treated leaves under laboratory conditions. Efficiency of the foregoing insecticides and their latent effect (larval duration, pupation and adult emergence) on treated larvae were studied as well. 
3.1. Field persistence of the tested insecticides residues and their efficiency against $S$. littoralis:

Persistence of the tested insecticides residues on cotton foliage under field conditions, expressed in $\mathrm{Lt}_{\mathbf{5 0}} \mathrm{s}$, and the corresponding fidutial limits and slope values are presented in Table 1 . Pyridalyl had significantly the superior persistent residual activity against $S$. littoralis $4^{\text {th }}$ instar larvae with $\mathrm{Lt}_{50}$ value of 6.74 days, followed by emamectin-benzoate with $\mathrm{Lt}_{50}$ value of 5.51 days. On the contrary, indoxacarb showed significantly the shortest persistence period translated in $\mathrm{Lt}_{\mathbf{5 0}}$ value of 0.72 day only, preceded by spinosad and profenofos with $\mathrm{Lt}_{\mathbf{5 0}}$ values of 1.33 and 1.55 days, respectively. This may be explained by either the diversity in cotton foliage capability for adsorption of different tested insecticides into epicuticular waxes and deeper leaf tissues or the degradation factors which act versus the persistence of insecticide residue on cotton foliage. Moreover, the persistence period of an insecticide residue, under the same weathering conditions, differs based on the used concentration, the sprayed plant and

Table 1. Persistence of the tested insecticides residues (Lethal time, $\mathrm{Lt}_{50}$ ) on cotton foliage under field conditions against $4^{\text {th }}$ instar larvae of Spodoptera littoralis field strain

\begin{tabular}{lccccc}
\hline \multicolumn{1}{c}{ Insecticide } & $\begin{array}{c}\text { Conc.* } \\
\text { mgAI/L }\end{array}$ & $\begin{array}{c}\mathbf{L t}_{\mathbf{5 0}} \\
\left(\mathbf{d a y s}^{\mathbf{n}}\right.\end{array}$ & $\mathbf{9 5 \% \text { Fidutial limits }}$ & Slope & $\boldsymbol{X}^{\mathbf{2}}$ \\
\hline Spinosad & 60 & 1.33 & $1.00-1.72$ & $1.04 \pm 0.111$ & 0.145 \\
\hline Emamectin-benzoate & 15 & 5.51 & $4.48-7.35$ & $1.73 \pm 0.222$ & 7.221 \\
\hline Pyridalyl & 250 & 6.74 & $5.11-8.23$ & $0.93 \pm 0.154$ & 3.989 \\
\hline Indoxacarb & 39 & 0.72 & $0.46-1.02$ & $0.79 \pm 0.102$ & 7.243 \\
\hline Profenofos & 2700 & 1.55 & $1.24-1.91$ & $1.35 \pm 0.129$ & 0.221 \\
\hline
\end{tabular}

*The used field recommended rate expressed in mg A.I. per $\mathrm{L}$

Table 2. Corrected mortality percentage and duration of survived $4^{\text {th }}$ instar larvae of Spodoptera littoralis field strain after feeding for $24 \mathrm{~h}$ on cotton leaves treated with various insecticides

\begin{tabular}{|c|c|c|c|c|c|c|c|c|c|c|c|}
\hline \multirow{3}{*}{ Treatment } & \multirow{3}{*}{$\begin{array}{l}\text { Conc. } \\
\text { mg AI } \\
\text { per L. }\end{array}$} & \multicolumn{10}{|c|}{ \%Mortality $(\% \mathrm{M})$ and larval duration(LD) at indicated days after spray: } \\
\hline & & \multicolumn{2}{|c|}{ Zero time } & \multicolumn{2}{|c|}{1 day } & \multicolumn{2}{|c|}{2 days } & \multicolumn{2}{|c|}{4 days } & \multicolumn{2}{|c|}{6 days } \\
\hline & & $\begin{array}{c}\% \mathrm{M}_{ \pm} \\
\mathrm{SD}\end{array}$ & $\begin{array}{c}\text { LD } \\
\text { days }\end{array}$ & $\begin{array}{c}\% \mathrm{M}_{ \pm} \\
\mathrm{SD}\end{array}$ & $\begin{array}{c}\text { LD } \\
\text { days }\end{array}$ & $\begin{array}{c}\% \mathrm{M}_{ \pm} \\
\mathrm{SD}\end{array}$ & $\begin{array}{c}\text { LD } \\
\text { days }\end{array}$ & $\begin{array}{c}\% \mathrm{M}_{ \pm} \\
\mathrm{SD}\end{array}$ & $\begin{array}{c}\text { LD } \\
\text { days }\end{array}$ & $\begin{array}{c}\% \mathrm{M}_{ \pm} \\
\text {SD }\end{array}$ & $\begin{array}{c}\text { LD } \\
\text { days }\end{array}$ \\
\hline Spinosad & 60 & $\begin{array}{l}86 \pm \\
2.35 \\
\end{array}$ & $18.6^{\mathrm{a}}$ & $\begin{array}{l}54 \pm \\
3.25 \\
\end{array}$ & $18.8^{\mathrm{a}}$ & $\begin{array}{l}43 \pm \\
2.57 \\
\end{array}$ & $19.1^{\mathrm{b}}$ & $\begin{array}{l}32 \pm \\
2.13 \\
\end{array}$ & $17.5^{\mathbf{b}}$ & $\begin{array}{l}24 \pm \\
1.78 \\
\end{array}$ & $16.0^{\mathrm{c}}$ \\
\hline $\begin{array}{c}\text { Emamectin- } \\
\text { benzoate }\end{array}$ & 15 & 100 & - & $\begin{array}{l}92 \pm \\
1.02\end{array}$ & - & $\begin{array}{l}88 \pm \\
2.01\end{array}$ & $21.2^{\mathrm{a}}$ & $\begin{array}{l}66 \pm \\
3.12 \\
\end{array}$ & $21.5^{\mathrm{a}}$ & $\begin{array}{l}41 \pm \\
2.11\end{array}$ & $19.6^{\mathrm{a}}$ \\
\hline Pyridalyl & 250 & 100 & - & 100 & - & $\begin{array}{l}95 \pm \\
0.87\end{array}$ & - & $\begin{array}{l}87 \pm \\
1.11\end{array}$ & $20.6^{\mathrm{a}}$ & $\begin{array}{l}72 \pm \\
1.58\end{array}$ & $20.1^{\mathrm{a}}$ \\
\hline Indoxacarb & 39 & $\begin{array}{l}65 \pm \\
2.56\end{array}$ & $19.5^{\mathrm{a}}$ & $\begin{array}{l}58 \pm \\
3.78\end{array}$ & $19.7^{\mathrm{a}}$ & $\begin{array}{l}35 \pm \\
2.31\end{array}$ & $20.6^{\mathrm{a}}$ & $\begin{array}{l}26 \pm \\
2.45 \\
\end{array}$ & $20.7^{\mathrm{a}}$ & $\begin{array}{l}14 \pm \\
1.99\end{array}$ & $18.2^{\mathbf{b}}$ \\
\hline Profenofos & 2700 & $\begin{array}{l}94 \pm \\
1.25\end{array}$ & - & $\begin{array}{l}60 \pm \\
4.12\end{array}$ & $15.2^{\mathrm{b}}$ & $\begin{array}{l}45 \pm \\
3.45\end{array}$ & $15.0^{\mathrm{c}}$ & $\begin{array}{l}30 \pm \\
2.98\end{array}$ & $15.6^{\mathbf{c}}$ & $\begin{array}{l}10 \pm \\
3.41\end{array}$ & $15.1^{\mathrm{c}}$ \\
\hline Control & - & 0.0 & $14.6^{\mathbf{b}}$ & 0.0 & $14.3^{\mathbf{b}}$ & 0.0 & $14.0^{\mathrm{c}}$ & 0.0 & $14.6^{\mathbf{c}}$ & 0.0 & $14.5^{\mathrm{c}}$ \\
\hline
\end{tabular}

- No survived larvae to complete larval stage.

Within a column, means followed by the same letter are not significantly different using DMRT $(\mathrm{P}<0.05)$.

$\%$ Mortality was estimated $48 \mathrm{~h}$ after treatment. 
Table 3. Pupation percentage of survived $4^{\text {th }}$ instar larvae of Spodoptera littoralis field strain after feeding for $24 \mathrm{~h}$ on cotton leaves treated with various insecticides

\begin{tabular}{|c|c|c|c|c|c|c|c|c|c|c|c|}
\hline \multirow{3}{*}{ Treatment } & \multirow{3}{*}{$\begin{array}{l}\text { Conc. } \\
\text { mgAI } \\
\text { per } \mathrm{L} .\end{array}$} & \multicolumn{10}{|c|}{ \%Pupation \pm SD at indicated days after spray: } \\
\hline & & \multicolumn{2}{|c|}{ Zero time } & \multicolumn{2}{|c|}{1 day } & \multicolumn{2}{|c|}{2 days } & \multicolumn{2}{|c|}{4 days } & \multicolumn{2}{|c|}{6 days } \\
\hline & & $\begin{array}{l}\text { \%nor. } \\
\text { pupae }\end{array}$ & $\begin{array}{l}\text { \%def. } \\
\text { pupae }\end{array}$ & $\begin{array}{l}\text { \%nor. } \\
\text { pupae }\end{array}$ & $\begin{array}{l}\text { \%def. } \\
\text { pupae }\end{array}$ & $\begin{array}{l}\text { \%nor. } \\
\text { pupae }\end{array}$ & $\begin{array}{l}\text { \%def. } \\
\text { pupae }\end{array}$ & $\begin{array}{l}\text { \%nor. } \\
\text { pupae }\end{array}$ & $\begin{array}{l}\text { \%def. } \\
\text { pupae }\end{array}$ & $\begin{array}{l}\text { \%nor. } \\
\text { pupae }\end{array}$ & $\begin{array}{l}\text { \%def } \\
\text { pupae }\end{array}$ \\
\hline Spinosad & 60 & $\begin{array}{l}39.8 \pm \\
2.3\end{array}$ & $\begin{array}{l}5.5 \pm \\
0.65\end{array}$ & $\begin{array}{l}58.9 \pm \\
3.1\end{array}$ & $\begin{array}{l}4.3 \pm \\
0.12\end{array}$ & $\begin{array}{l}73.1 \pm \\
3.6\end{array}$ & $\begin{array}{l}3.5 \pm \\
0.21\end{array}$ & $\begin{array}{l}79.9 \pm \\
2.9\end{array}$ & $\begin{array}{l}3.8 \pm \\
0.19\end{array}$ & $\begin{array}{l}85.7 \pm \\
4.5\end{array}$ & $\begin{array}{l}3.2 \pm \\
0.05 \\
\end{array}$ \\
\hline $\begin{array}{l}\text { Emamectin- } \\
\text { benzoate }\end{array}$ & 15 & - & - & - & - & $\begin{array}{l}37.3 \pm \\
2.5\end{array}$ & $\begin{array}{l}15.6 \pm \\
1.25\end{array}$ & $\begin{array}{l}38.4 \pm \\
2.6\end{array}$ & $\begin{array}{l}13.9 \pm \\
1.12\end{array}$ & $\begin{array}{l}42.6 \pm \\
1.5\end{array}$ & $\begin{array}{l}10.3 \pm \\
1.22\end{array}$ \\
\hline Pyridalyl & 250 & - & - & - & - & - & - & $\begin{array}{l}12.2 \pm \\
1.1\end{array}$ & $\begin{array}{l}10.7 \pm \\
1.36\end{array}$ & $\begin{array}{l}20.4 \pm \\
2.4\end{array}$ & $\begin{array}{l}12.9 \pm \\
1.87\end{array}$ \\
\hline Indoxacarb & 39 & $\begin{array}{l}26.4 \pm \\
3.4\end{array}$ & $\begin{array}{l}20.3 \pm \\
1.23 \\
\end{array}$ & $\begin{array}{l}45.7 \pm \\
1.5\end{array}$ & $\begin{array}{l}16.8 \pm \\
1.5 \\
\end{array}$ & $\begin{array}{l}49.2 \pm \\
2.8\end{array}$ & $\begin{array}{l}10.9 \pm \\
0.28\end{array}$ & $\begin{array}{l}61.5 \pm \\
3.2\end{array}$ & $\begin{array}{l}8.4 \pm \\
0.89 \\
\end{array}$ & $\begin{array}{l}73.3 \pm \\
4.2\end{array}$ & $\begin{array}{l}5.4 \pm \\
0.45 \\
\end{array}$ \\
\hline Profenofos & 2700 & - & - & $\begin{array}{l}72.4 \pm \\
3.4\end{array}$ & $\begin{array}{l}5.6 \pm \\
1.1 \\
\end{array}$ & $\begin{array}{l}76.8 \pm \\
2.9 \\
\end{array}$ & $\begin{array}{l}4.9 \pm \\
0.36\end{array}$ & $\begin{array}{l}85.2 \pm \\
4.1\end{array}$ & $\begin{array}{l}3.4 \pm \\
0.67 \\
\end{array}$ & $\begin{array}{l}90.4 \pm \\
3.4\end{array}$ & $\begin{array}{l}3.1 \pm \\
0.57 \\
\end{array}$ \\
\hline Control & - & $\begin{array}{l}96.2 \pm \\
1.6\end{array}$ & $\begin{array}{l}2.3 \pm \\
0.12\end{array}$ & $\begin{array}{l}97.1 \pm \\
1.1\end{array}$ & $\begin{array}{l}1.8 \pm \\
0.31\end{array}$ & $\begin{array}{l}96.8 \pm \\
1.4\end{array}$ & $\begin{array}{l}1.3 \pm \\
0.05\end{array}$ & $\begin{array}{l}97.5 \pm \\
1.2\end{array}$ & $\begin{array}{l}1.2 \pm \\
0.09\end{array}$ & $\begin{array}{l}96.3 \pm \\
1.7\end{array}$ & $\begin{array}{l}2.1 \pm \\
0.32\end{array}$ \\
\hline
\end{tabular}

- No survived larvae to develop into pupae.

Percentage of normal pupae (\%nor. pupae) and deformed pupae (\%def. pupae) were calculated based on the number of survived larvae $48 \mathrm{~h}$ after treatment.

Table 4. Adult emergence percentage of survived $4^{\text {th }}$ instar larvae of Spodoptera littoralis field strain after feeding for $24 \mathrm{~h}$ on cotton leaves treated with various insecticides

\begin{tabular}{|c|c|c|c|c|c|c|c|c|c|c|c|}
\hline \multirow{3}{*}{ Treatment } & \multirow{3}{*}{$\begin{array}{l}\text { Conc. } \\
\text { mg AI } \\
\text { per L. }\end{array}$} & \multicolumn{10}{|c|}{ \%Adult emergence \pm SD at indicated days after spray: } \\
\hline & & \multicolumn{2}{|c|}{ Zero time } & \multicolumn{2}{|c|}{1 day } & \multicolumn{2}{|c|}{2 days } & \multicolumn{2}{|c|}{4 days } & \multicolumn{2}{|c|}{6 days } \\
\hline & & $\begin{array}{l}\% \text { nor. } \\
\text { adults }\end{array}$ & $\begin{array}{l}\text { \%malf. } \\
\text { adults }\end{array}$ & $\begin{array}{l}\% \text { nor. } \\
\text { adults }\end{array}$ & $\begin{array}{c}\% \text { malf. } \\
\text { adults }\end{array}$ & $\begin{array}{c}\% \text { nor. } \\
\text { adults }\end{array}$ & $\begin{array}{c}\% \text { malf. } \\
\text { adults }\end{array}$ & $\begin{array}{c}\% \text { nor. } \\
\text { adults }\end{array}$ & $\begin{array}{c}\% \text { malf. } \\
\text { adults }\end{array}$ & $\begin{array}{l}\% \text { nor. } \\
\text { adults }\end{array}$ & $\begin{array}{c}\text { \% malf. } \\
\text { adults }\end{array}$ \\
\hline Spinosad & 60 & $\begin{array}{l}52.1 \pm \\
2.9\end{array}$ & $\begin{array}{l}10.6 \pm \\
0.89\end{array}$ & $\begin{array}{l}64.3 \pm \\
2.6\end{array}$ & $\begin{array}{l}6.7 \pm \\
0.55\end{array}$ & $\begin{array}{l}79.5 \pm \\
3.5\end{array}$ & $\begin{array}{l}5.2 \pm \\
0.12\end{array}$ & $\begin{array}{l}87.1 \pm \\
4.2\end{array}$ & $\begin{array}{l}3.3 \pm \\
0.32\end{array}$ & $\begin{array}{l}93.3 \pm \\
4.6\end{array}$ & $\begin{array}{l}2.6 \pm \\
0.23\end{array}$ \\
\hline $\begin{array}{l}\text { Emamectin- } \\
\text { benzoate }\end{array}$ & 15 & - & - & - & - & $\begin{array}{l}35.9 \pm \\
2.1\end{array}$ & $\begin{array}{l}17.7 \pm \\
1.4\end{array}$ & $\begin{array}{l}39.8 \pm \\
2.7\end{array}$ & $\begin{array}{l}15.4 \pm \\
2.21\end{array}$ & $\begin{array}{l}55.2 \pm \\
2.6\end{array}$ & $\begin{array}{l}12.3 \pm \\
0.54\end{array}$ \\
\hline Pyridalyl & 250 & - & - & - & - & - & - & $\begin{array}{l}21.3 \pm \\
1.6 \\
\end{array}$ & $\begin{array}{l}13.8 \pm \\
0.98 \\
\end{array}$ & $\begin{array}{l}26.7 \pm \\
2.4 \\
\end{array}$ & $\begin{array}{l}14.2 \pm \\
1.45 \\
\end{array}$ \\
\hline Indoxacarb & 39 & $\begin{array}{l}55.3 \pm \\
2.8\end{array}$ & $\begin{array}{l}14.7 \pm \\
1.05\end{array}$ & $\begin{array}{l}61.4 \pm \\
3.1\end{array}$ & $\begin{array}{l}12.2 \pm \\
0.52\end{array}$ & $\begin{array}{l}72.6 \pm \\
4.6\end{array}$ & $\begin{array}{l}10.4 \pm \\
0.91\end{array}$ & $\begin{array}{l}76.3 \pm \\
3.4\end{array}$ & $\begin{array}{l}7.5 \pm \\
0.75\end{array}$ & $\begin{array}{l}81.4 \pm \\
4.5\end{array}$ & $\begin{array}{l}5.6 \pm \\
0.12\end{array}$ \\
\hline Profenofos & 2700 & - & - & $\begin{array}{l}72.1 \pm \\
2.9\end{array}$ & $\begin{array}{l}5.2 \pm \\
0.11 \\
\end{array}$ & $\begin{array}{l}80.5 \pm \\
3.6\end{array}$ & $\begin{array}{l}3.4 \pm \\
0.44 \\
\end{array}$ & $\begin{array}{l}85.7 \pm \\
3.1 \\
\end{array}$ & $\begin{array}{l}3.1 \pm \\
0.31 \\
\end{array}$ & $\begin{array}{l}95.2 \pm \\
2.4\end{array}$ & $\begin{array}{l}2.4 \pm \\
0.33 \\
\end{array}$ \\
\hline Control & - & $\begin{array}{l}97.2 \pm \\
1.5\end{array}$ & $\begin{array}{l}1.4 \pm \\
0.22\end{array}$ & $\begin{array}{l}98.3 \pm \\
1.4\end{array}$ & $\begin{array}{l}1.8 \pm \\
0.06\end{array}$ & $\begin{array}{l}97.7 \pm \\
1.5\end{array}$ & $\begin{array}{l}2.2 \pm \\
0.32\end{array}$ & $\begin{array}{l}97.9 \pm \\
0.9\end{array}$ & $\begin{array}{l}1.2 \pm \\
0.91\end{array}$ & $\begin{array}{l}97.5 \pm \\
1.5\end{array}$ & $\begin{array}{l}0.9 \pm \\
1.11\end{array}$ \\
\hline
\end{tabular}

- No survived larvae to develop into adults.

Percentage of normal adults (\%nor. adults) and malformed adults (\%malf. adults) were calculated based on the total number of pupae (normal and deformed).

These results substantiated the abovementioned high persistence of pyridalyl and emamectin-benzoate residues on cotton foliage and their unique and/or new modes of action which translated in low insect resistance. This may be due to pyridalyl and emamectinbenzoate both are not frequently used against $S$. littoralis in Egypt and relatively new compounds in the Egyptian market of insecticides.

On the other hand, the efficiency of the conventional insecticide, profenofos, declined sharply and significantly 2 days post spray recording $94,60,45,30$, and $10 \%$ larval mortality after $0,1,2,4$, and 6 days of application, respectively. In this respect, indoxacarb and spinosad exhibited the inferior activity at the beginning of the experiment; then decreased gradually till the experiment end. Cook et al. (2004) found that pyridalyl and emamectin-benzoate controlled $S$. exigua infestation up to 10 days after treatment of cotton field- 
plants compared to untreated control. Emamectinbenzoate proved to be more efficient than spinosad and indoxacarb against $S$. litura larvae (Ahmad et al., 2006). The field recommended rate of methylamine avermectin (compound of the same group of emamectin-benzoate) caused $100,93.6,81.7,68.4$, and $51.6 \%$ mortality of $S$. littoralis $2^{\text {nd }}$ instar larvae after $0,1,2,4$, and 7 days of spraying cotton foliage, respectively under field conditions (Dahi et al., 2009). Moreover, Abdu-Allah (2010) stated that after spraying of cotton field-plants, the efficiency of emamectin-benzoate against $4^{\text {th }}$ instar larvae of $S$. littoralis decreased gradually and significantly recording $98.00,70.00,36.67$, and $0.00 \%$ mortality after $0,3,6$, and 10 days of application, respectively.

\subsection{Latent effects of the tested insecticides on $S$. littoralis:}

Most of previous studies about the efficiency of novel insecticides on lepidopteran pests had been conducted on larval stages and little has been published about their latent effects on pupae and adults. In this study, data presented in Table 2 indicated that emamectin-benzoate significantly prolonged the larval duration (21.5 days) comparing to control treatment (14.6 days) when the larvae were fed on sprayed leaves after 4 days of application; followed by indoxacarb (20.7 days), pyridalyl (20.6 days) and spinosad (17.5 days).While, profenofos did not cause significant change in larval duration (15.6 days). As the time after spray extended, the effect of all tested compounds on larval duration declined. Similar results were found by AbdelRahim et al. (2009); they mentioned that pyridalyl significantly prolonged larval duration (18.0 days) of $S$. littoralis field strain, followed by spinosad (12.5 days) and methylamine avermectin (12.3days) comparing to control (8.8 days) when these compounds were applied at their $\mathrm{LC}_{\mathbf{5 0}}$ values against $4^{\text {th }}$ instar larvae.

As criteria of latent effects, percentages of normal pupae, deformed pupae, normal adults and malformed adults were studied and the obtained data are discussed in Tables 3 and 4. It is noticed that, no survived larvae to develop into pupae in treatments which were fed on sprayed leaves with pyridalyl, emamectin-benzoate and profenofos after 2 days, 1 day, and zero time of spray, respectively. It is also remarkable that, the negative effect of the tested insecticides on percentages of pupation and adult emergence decreased as the time after spray increased. Larvae which were fed on indoxacarb-treated leaves after zero time and one day of spray significantly produced the least percentage of normal pupae (26.4 and 45.7\%) and the highest percentage of deformed pupae (20.3 and 16.8\%), but this effect subsequently dropped. After 6 days of spray, pyridalyl and emamectin-benzoate demonstrated the highest persistent negative effect on pupation causing $20.4 \& 42.6 \%$ normal pupae and $12.9 \& 10.3 \%$ deformed pupae, respectively comparing to the other treatments. The conventional insecticide, profenofos, had feeble effect on normal pupation. The same direction of act was observed regarding latent effect on adult emergence (Table 4). When the larvae were fed on sprayed leaves after 6 days of application, pyridalyl and emamectin-benzoate significantly resulted in the highest percentage of malformed adults (14.2 and 12.3\%) and the lowest percentage of normal adults (26.7 and $55.2 \%$ ), respectively comparing to all other tested insecticides and the control. Abdel-Rahim et al. (2009) stated that pyridalyl and spinosad at their $\mathrm{LC}_{\mathbf{5 0}}$ values against $4^{\text {th }}$ instar of $S$. littoralis field strain resulted in $60.7 \& 68.3 \%$ normal pupae and $74 \& 72 \%$ normal adults. Also, they reported that the percentages of deformed pupae and malformed adults were the highest with pyridalyl application (10.8 and $22.0 \%$ ) comparing to spinosad application (2.2 and 3.3\%), respectively. Osman and Mahmoud (2009) found that spinosad at its recommended rate against $3^{\text {rd }}$ instar larvae of S. littoralis susceptible strain resulted in $33.3 \%$ pupation, $13.3 \%$ adult emergence and $6.6 \%$ malformed adults.

\section{CONCLUSION}

The obtained results of this study revealed that the novel insecticides, pyridalyl and emamectin-benzoate, had high persistent residues on cotton plants under field conditions. In addition, they demonstrated the superior effectiveness against the cotton leaf worm, Spodoptera littoralis, for the longest periods post application causing high initial and latent effects comparing to the conventional insecticides. Moreover, many previous studies clarified that pyridalyl and emamectin-benzoate exhibited low toxicity to natural enemies and environment components. Thus, these novel insecticides represent an important choice for use in Integrated Pest Management programs as substitutes of conventional insecticides to control the cotton leaf worm, particularly under field conditions.

\section{REFERENCES}

Abbott, W. S., 1925. A method of computing the effectiveness of an insecticide. J. Econ. Entomol., 18: 265-267.

Abdel-Rahim, E.F.; A.M. Azab; M.M. Ali; G.A. Morsi and M.A. Ahmad, 2009. Comparative toxicity of three novel biotic compounds, spinosad, pyridalyl and radical in relative to a conventional insecticide, lannate against the field and laboratory strains of the second and fourth instar larvae of cotton leaf worm Spodoptera littoralis (Boisd.). Egypt. J. Agric. Res., 87(2): 433 - 453.

Abdu-Allah, G.A.M., 2010. Laboratory and field evaluation of emamectin benzoate and spinetoram on cotton leafworm larvae. Resis. Pest Manag. Newsletter, 20(1): 13-17. 
Abo-Elghar, G.E.; Z.A. Elbermawy; A.G. Yousef and H.K. Abd Elhaddy, 2005.Monitoring and characterization of insecticide resistance in the cotton leafworm, Spodoptera littoralis (Lepidoptera: Noctuidae). J. Asia. Pacific Entomol., 8: 397-410.

Ahmad, M.; M.A. Saleem; M. Ahmad and A.H. Sayyed, 2006. Time trends in mortality for conventional and new insecticides against leaf worm, Spodoptera litura, (Lepidoptera: Noctuidae). Pakistan J. Biolog. Sci., 9(3): $360-364$.

Argentine, J.A.; R.K. Jansson; W.R. Halliday; D. Rugg and C.S. Jany, 2002. Potency, spectrum and residual activity of four new insecticides under glasshouse conditions. Florida Intomologist, 85: 552-562.

Aydin, M.H. and M.O. Gürkan, 2006. The efficacy of spinosad on different strains of Spodoptera littoralis (Boisduval) (Lepidoptera: Noctuidae). Turk. J. Biol., 30: 5-9.

Azab, S.G.; M.M. Sadek and K. Crailsheim, 2001. Protein metabolism in larvae of the cotton leafworm, Spodoptera littoralis (Lepidoptera: Noctuidae) and its response to three mycotoxins. J. Econ. Entomol. 30: 817-823.

Brown, E.S. and C.F. Dewhurst, 1975. The genus Spodoptera (Lepidoptera: Noctuidae) in Africa and the Near East. Bull. Entomol. Res., 65:21-61.

Cook, D.R.; B.R. Leonard and J. Gore, 2004. Field and laboratory performance of novel insecticides against armyworms (Lepidoptera: Noctuidae). Florida Entomologist, 87(4): 433 - 439.

CoStat Program, 2006. Version 6.311, Cohort Software Inc., Monterey http: // www.cohort.com/download.costate. html.

Dahi, H.F.; Y.A. El-Sayed; N.M. El-Barkey and M.F. AbdElaziz, 2009. Toxicological and biochemical studies of methylamine Avermectin, a new type of bioinsecticide against the cotton leaf worm, Spodoptera littoralis (Boisd.). Egypt Acad. J. Biolog. Sci., 2(1): 103 - 116.

El-Barkey, N.M.; H.F. Dahi and Y.A. El- Sayed, 2008. Toxicological evaluation and biochemical impacts for radiant as a new generation of Spinosyn on Spodoptera littoralis (Boisd.) larvae. Egypt Acad.J. Biolog. Sci., 1: 85 $-97$.

El-Defrawi, M.E.; A. Toppozada; N. Mansour and M. Zeid, 1964. Toxicological studies on the Egyptian cotton leafworm, Pordenia litura (F.). 1. Susceptibility of different larval instar of $P$. litura to insecticides, J. Econ. Entomol., 57: 591-593.

Finney, J.D., 1971. Probit analysis ( $3^{\text {rd }}$ Edn.) Cambridge University Press. UK.
Foster, S.P.; I. Denholm and R. Thompson, 2003. Variation in response to Neonicotinoid insecticides in peach-potato aphids, Myzus persicae (Homoptera: Aphididae). Pest Manag. Sci., 59(2):166-173.

Gupta, S.; R.K. Sharma and R.K. Gupta, 2009. Persistence of new insecticides and their efficacy against insect pests of okra. Bull. Environ. Contam. Toxicol., 82: 243 - 247.

Holland, P.T.; T.K. McGhie and C.P. Malcolm, 1984. Residual life of pesticides on kiwifruit. Proc. $37^{\text {th }}$ N.Z. Weed and Pest Cont. Conf.:136- 141.

Ishaaya, I.; S. Kontsedalov and A. Horowitz, 2002. Emamectin, a novel insecticide for controlling field crop pests. Pest Manage. Sci., 58(11): 1091- 1095.

Le Ora Software, 1987. POLO-PC, A User Guide to Probit or Logit Analysis, Le Ora Software, Berkeley, CA, USA.

McDonald, P.T.; M.K. Kish; P.A. King; F.J. Dunagan and R.T. Weiland, 1998. Field persistence of several insecticides on cotton foliage as determined by beet armyworm Spodoptera exigua bioassay. Proceedings Beltwide Cotton Conf. 2: 1164-1166.

Michaud, J.P. and A.K. Grant, 2003. IPM-compatibility of foliar insecticides for citrus: indices derived from toxicity to beneficial insects from four orders. J. Insect Sci., 3: 110 .

Osman, M.A.M. and M.F. Mahmoud, 2009. Effects of biorational insecticides on selected biological aspects of the Egyptian cotton leaf worm Spodoptera littoralis (Boisd.) (Lepidoptera: Noctuidae). J. Plant Prot. Res., 49(2): 135 140.

Pineda, S.; M.I. Schneider; G. Smagghe; A.M. Martinez; P.D. Estal; E. Vinuela; J. Valle and F. Budia, 2007. Lethal and sublethal effects of methoxyfenozide and spinosad on Spodoptera littoralis (Lepidoptera: Noctuidae). J. Econ. Entomol., 100(3): 773-780.

Sakamoto, N.; N. Ufdo; K. Umeda; S. Matsuot; T. Hagat; T. Fujisawa and Y. Tomigahara, 2005. Research and Development of a Novel Insecticide 'Pyridalyl' "Sumitomo Kagaku", Chem. Abstr., (1): 33-44.

Smagghe, G.; B. Carton; W. Wesemael; I. Ishaaya and L. Tirry,1999. Ecdysone agonists-mechanism of action and application on Spodoptera species. Pestic. Sci., 55: 343389.

Smagghe, G.; S. Pineda; B. Carton; P.D. Estal; F. Budia and E. Vinuela, 2003. Toxicity and kinetics of methoxyfenozide in greenhouse-selected Spodoptera exigua (Lepidoptera: Noctuidae). Pest Manag. Sci., 59: 1203-1209.

SPSS, 2004. SPSS 13.0 for Windows, SPSS Inc., Chicag.

Thompson, G.D.; R. Dutton and T.C. Sparks, 2000. Spinosada case study: an example from natural products discovery programme. Pest Manag. Sci., 56: 696-702. 


\section{الملخص العربي}

\section{الثبات الحقلي لمتبقيات بعض المبيدات الحديثة على نبات القطن وتأثيراتما المتأخرة}

\section{علي دودة ورق القطن Spodoptera littoralis (Boisduval)}

$$
\begin{aligned}
& \text { الزاهي صابر الزاهي } \\
& \text { بروفينوفوس هي الأقل ثبـاتا مسسجلة قيم Lt50 هي } \\
& \text { 1.33، } 1.53 \text { يوم على الترتيب. }
\end{aligned}
$$

